\title{
Using the Mathematical Criterion of Gas Pipeline Failure to Determine the Remaining Operation Life
}

\author{
Minsariya Bayburova ${ }^{1}$, Zulfiya Ismagilova ${ }^{2}$ \\ ${ }^{1}$ Candidate of engineering sciences, associate professor, associate professor of the oil and gas transportation and storage department, \\ Almetyevsk State Oil Institute, RU-423450, Tatarstan, Almetyevsk, Russia \\ ${ }^{2}$ Candidate of engineering sciences, associate professor, associate professor of the gas and oil pipe and storage construction and repair \\ department, Ufa State Petroleum Technological University, RU-450064, Ufa, Republic of Bashkortostan, Russia
}

\begin{abstract}
During the years of operation, underground pipelines deteriorate because of aging, wear, damage, and destruction. A comprehensive inspection of the main pipeline revealed various defects on a certain pipeline route section. To identify the remaining operation life of the pipeline, a number of hydraulic tensile strength tests were performed using the cyclic loading method. The authors propose to calculate the minimum allowable wall thickness capable of withstanding the maximum internal pressure, as an alternative to cyclic hydraulic tests. The strength calculations were performed using the well-known mathematical strength criteria proposed by K.V. Zakharov and I.I. Goldenblatt and V.A. Kopnov.
\end{abstract}

Keywords: oil and gas pipeline, hydraulic testing, pipeline diagnostics, cyclic tests, mathematical strength criterion

\section{Introduction}

Providing reliable and trouble-free operation of main pipelines transporting crude oil and oil products is an important issue. Pipelines used in oil and gas fields deteriorate over time due to aging, wear, damage, and destruction. Such deterioration can be caused by both corrosive and non-corrosive factors.

The problem of stress corrosion cracking of pipe steels is one of the most urgent in countries with an extensive system of underground gas pipelines.

The development of methods for predicting the state of the linear part of main gas pipelines today is one of the priority areas in the oil and gas industry. The issues of diagnostics and monitoring of stress corrosion cracking are covered in the works of Russian and foreign scientists and other specialists in the field of stress corrosion cracking [1,2,3]. However, methods for assessing the tendency of sections of the linear part of gas pipelines to stress corrosion cracking require further research and development.

Features of operation and reliability of pipelines transporting hydrogen sulfide-containing media are reflected in the works of scientists $[4,5]$.

Brittle fractures can occur in ductile steel structures at stresses well below the yield strength. The fractures can be caused by defects such as tiny cracks that may occur during steelmaking or appear and develop during the structure operation. Therefore, steel structures require timely diagnostics. Such diagnostics can be performed using modern non-destructive testing methods that help detect cracks and other defects in the course of operation [4].

At the same time, it is important to take into account that the hydraulic tests carried out are quite laborious. To reduce material costs and obtain the desirable result, it can be sufficient to calculate the minimum permissible wall thickness using a phenomenological method for this, considering the pipe material as an averaged solid material - homogeneous anisotropic.

\section{Results and discussion}

Pipeline transport requires constant engineering maintenance to ensure safe operation and a certain level of quality. The need to keep pipelines in good condition requires researchers to look for new effective methods of pipe inspection in order to detect defects and cracks, as well as corrosion on their surface.

Non-destructive testing as a means of detecting signs of destruction of pipeline material and preventing emergencies is relevant today. Methods for determining the dimensions and orientation of defects are constantly developing, equipment is being improved, research and testing are carried out to identify the characteristics of flaw detector models, and analysis is performed to further improve the operation of flaw detection tools.

A comprehensive inspection of the gas pipeline revealed various defects on a certain pipeline route section. The main defects observed were bulges, blisters, and delaminations in the pipe (Figure 1). 


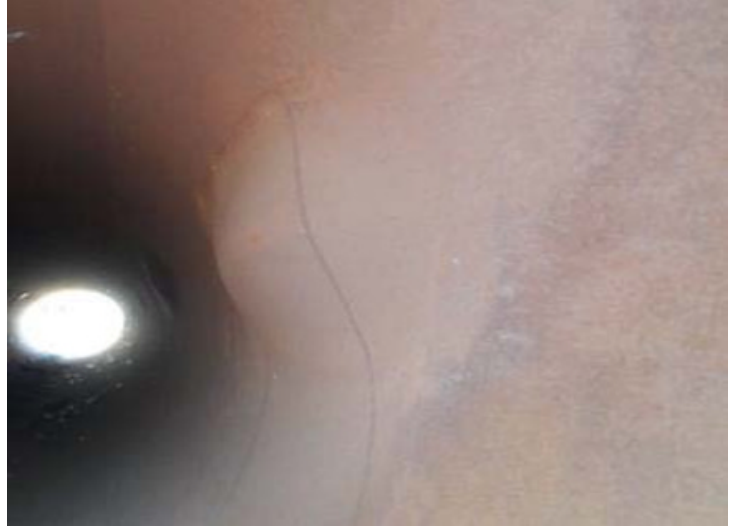

Fig. 1. Detected blister-type defect

The pipes are made of low-carbon low-alloy steel with a $0.18 \ldots . .0 .20 \%$ carbon content, a tensile strength of at least $520 \mathrm{MPa}$, yield strength of at least $300 \mathrm{MPa}$, and impact strength of $500 \mathrm{~kJ} / \mathrm{m}^{2}$ at minus $40^{\circ} \mathrm{C}$. The pipes are made of normalised sheets.

Main design specifications of the section under consideration: design pressure $-5.4 \mathrm{MPa}$, allowable operating pressure - $3.4 \mathrm{MPa}$, pipeline diameter $1,020 \mathrm{~mm}$, and wall thickness - 14-16 mm.

To study the behavior of the detected gas pipeline defects during the subsequent operation, a number of tensile strength tests were performed using the cyclic loading method. Pipe samples taken from the linear part of the pipelines with a defect on the internal surface were made in the form of barrels and equipped with plugs (Fig. 2).

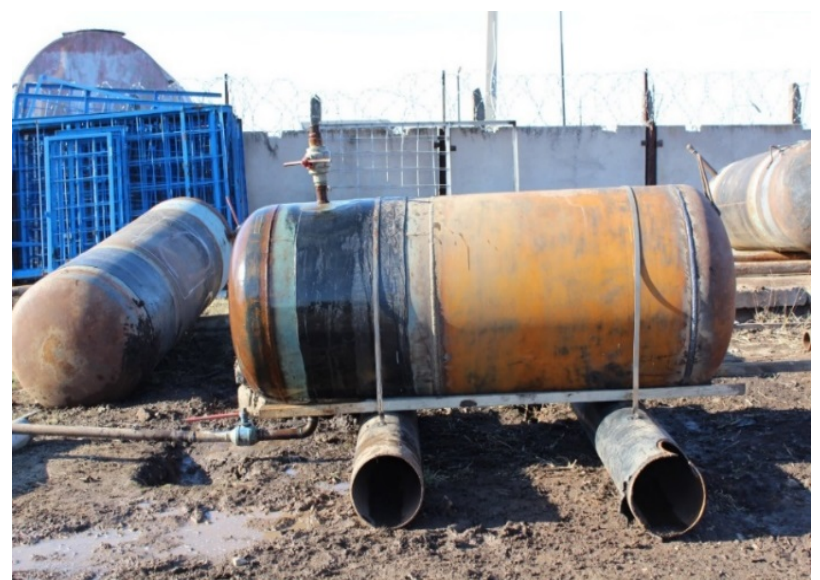

Fig. 2. A sample barrel before hydraulic testing

Samples were cut out both along the rolling direction and crosswise. An additional pipeline system with a pressure gauge and a tap to measure cyclic and destruction parameters was installed on the test barrel at a safe distance.

The pressure was initially increased to $6 \mathrm{MPa}$ using a hydraulic pump; after 24 hours it was lowered to $5 \mathrm{MPa}$; then after another 24 hours - to $4 \mathrm{MPa}$, etc.

At

the next stage, the pressure was increased to $7 \mathrm{MPa}$ and the test was repeated. After testing at $8 \mathrm{MPa}$ and $9 \mathrm{MPa}$, the sample was destroyed by a pressure of 9.5 MPa (Fig. 3).
The tests proved that, despite the identified defects, the pipe's properties remained unchanged, so it could remain operational at the permitted operating pressure of 3.4 MPa;

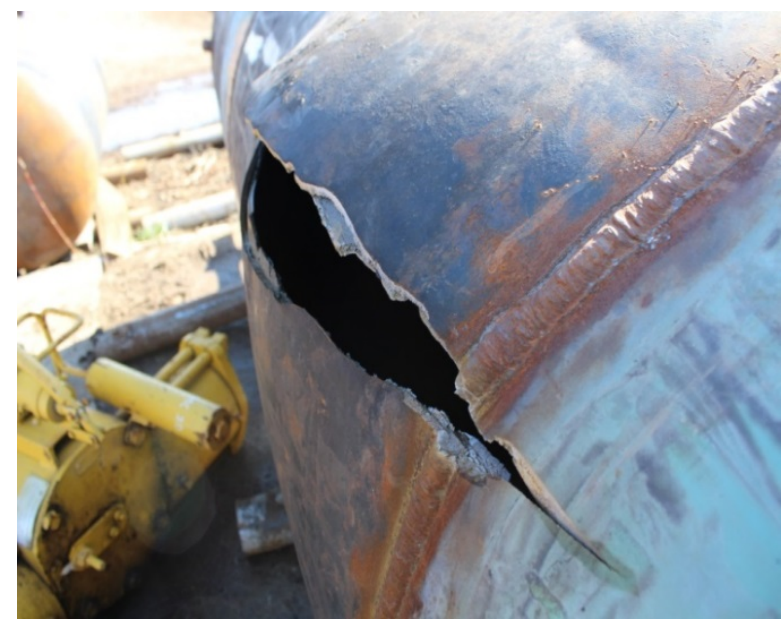

Fig. 3. Destruction of the barrel after hydraulic testing

The tests showed that the tensile strength values lengthwise and crosswise the rolling direction are consistent with the certified pipe material parameters. The same result was obtained by the authors theoretically. It was recognised that there was a metallurgical defect that could be classified as a delamination along the lap formed during the pipe piercing and rolling. Besides, the assumption was made that the damage may have been caused by growing propagation of the metallurgical defect (expanding discontinuities) spread across the entire wall thickness in the course of the gas pipeline operation when exposed to gas pressure and stresses created by low-cycle loads.

Delaminations were observed on the barrel surface after hydraulic testing (Fig. 4). The testing revealed delamination on the surface of the samples cut from used pipes. No delamination was observed in emergency stock pipes.

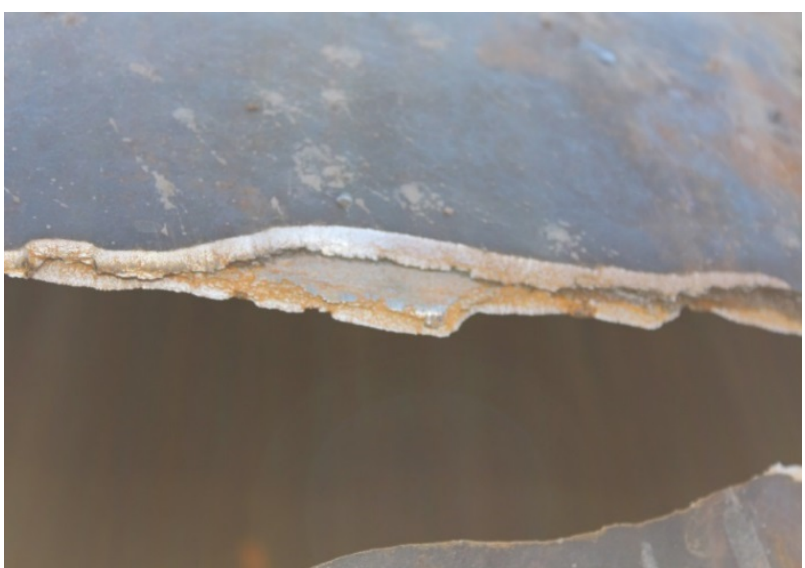

Fig. 4. Delamination of the barrel surface after hydraulic testing

The test results indicate that the cold gas transported by the pipe affects the state of its wall surface and leads to blistering and delamination of the latter. The reason

* Corresponding author: 
for the appearance of such blistering and delamination can be explained by the condition of the gas pipeline operation at the stage when the extracted raw gas is supplied directly to the gas pipeline. A sharp change in gas pressure (from the initial pressure of 12-13 $\mathrm{MPa}$ to the operating pressure) leads to a decrease in the gas temperature and a change in the stress state of the pipe material. The temperature jump of the inner and outer surfaces of the pipe reaches $60^{\circ} \mathrm{C}$, which in turn leads to the delamination of the pipe steel structure (Fig. 5). For this reason, a delamination-prone pipeline cannot provide the operability envisaged by the technical design.

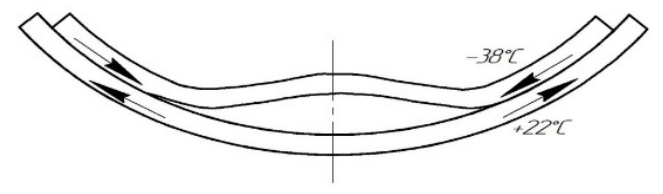

Fig. 5. Pipe wall buckling failure diagram

The hydraulic tests performed are costly and timeconsuming. The detected pipe blisters formed as a result of rolling defects or defects that occurred in the course of operation (for example, internal corrosion) could cause a decrease in the wall thickness. Therefore, the allowable wall thickness capable of withstanding the internal operating pressure must be obtained to prevent pipe rupture.

In [6], the anisotropy of mechanical characteristics was revealed as a result of tests performed on flat samples cut out from dismantled pipes (samples were cut along and across the rolling direction).

Considering that metal sheets after rolling or upsetting have orthotropic properties and assuming that pipe steel may be characterised by anisotropic elasticity [6], the minimum allowable wall thickness can be calculated with the use of the strength criteria for orthotropic materials, without performing a tensile test [7].

In order to determine the most effective criterion, it is necessary to analyse the applicability of the criterion for the material used, and it is also necessary to take into account that the mathematical expression of the accepted strength condition is as simple as possible. As an example, we will illustrate the method of selecting the desired criterion. For comparison purposes, we can take two commonly used strength criteria for anisotropic rocks - Zakharov and Goldenblatt-Kopnov criteria [8, 9] — and then use the most suitable one for a particular case.

When assessing the strength of an orthotropic material, its characteristics are determined in the direction of the orthotropic axes and at an angle of 45 degrees to them (Figure 6). The Goldenblatt-Kopnov model takes into account the ultimate shear strength of a material rotated by 45 degrees at positive and negative signs of shear stresses [7].

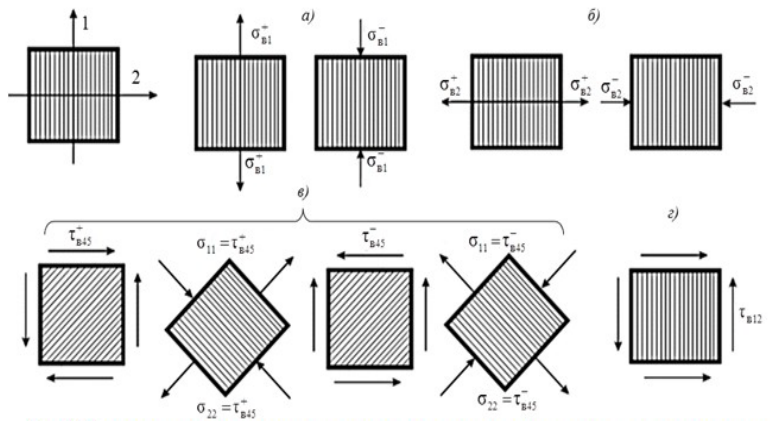

Fig. 6. Diagram of the main experiments to determine the components of the surface tensor for the Goldenblatt-Kopnov strength criterion.

a) $\sigma_{\mathrm{B}_{1}}^{+}$and $\sigma_{\mathrm{B}_{1}}^{-}$are ultimate tensile and compressive strengths in the direction of axis 1 ;

b) $\sigma_{\mathrm{B}_{2}}^{+}$and $\sigma_{\mathrm{B}_{2}}^{-}-$ultimate tensile and compressive strengths in the direction of axis 2;

c) $\tau_{\mathrm{B}_{45}}^{+}$и $\tau_{\mathrm{B}_{45}}^{-} \quad-$ ultimate shear strengths along areas inclined to the main ones at an angle of 45 degrees;

d) $\tau_{\mathrm{B}_{1,2}}$ - shear strength at the site with axes 1 and 2 .

In developing his criterion, K.V.Zakharov limited himself only to a coordinate system that coincides with the axes of symmetry of the mechanical properties of the material (5). Zakharov writes the strength condition for materials with different tensile and compressive strength as a second-order surface equation:

$$
\begin{gathered}
a_{1} \sigma_{x}^{2}+a_{2} \sigma_{y}^{2}+a_{3} \sigma_{z}^{2}+a_{4} \sigma_{x} \sigma_{y}+a_{5} \sigma_{x} \sigma_{z}+a_{6} \sigma_{y} \sigma_{z}+ \\
+a_{7} \sigma_{x}+a_{8} \sigma_{y}+a_{9} \sigma_{z}+a_{10} \tau_{x y}^{2}+a_{11} \tau_{x z}^{2}+a_{12} \tau_{y z}^{2}+ \\
+a_{13}=0
\end{gathered}
$$

In the case of a plane stress state, the strength condition in the principal stresses is as follows:

$$
\sigma_{1}^{2}+k_{1} \sigma_{1}^{2}+k_{2} \sigma_{1} \sigma_{2}+k_{3} \sigma_{1}+k_{4} \sigma_{2}+k_{5}=0,
$$

where $a_{1}, a_{2}, k_{1}, k_{2} \ldots-$ are constant factors that determine the strength of materials under different loading conditions.

After expressing the factors in terms of the corresponding tensile strength, the criterion (2) looks like [5]

$$
\begin{gathered}
\sigma_{1}^{2}+\frac{\sigma_{1 p} \sigma_{1 c}}{\sigma_{2 p} \sigma_{2 c}} \sigma_{2}^{2}+\left[1+\frac{\sigma_{1 p} \sigma_{1 c}}{\sigma_{2 p} \sigma_{2 c}}+\frac{\sigma_{1 c}-\sigma_{1 p}}{\tau_{45}}-\right. \\
\left.-\frac{\sigma_{1 p} \sigma_{1 c}}{\sigma_{2 p} \sigma_{2 c}}\left(\frac{\sigma_{2 c}-\sigma_{2 p}}{\tau_{45}}\right)-\frac{\sigma_{1 p} \sigma_{1 c}}{\tau_{45}^{2}}\right] \cdot \sigma_{1} \sigma_{2}+\left(\sigma_{1 c}-\sigma_{1 p}\right) \sigma_{1}+ \\
+\frac{\sigma_{1 p} \sigma_{1 c}}{\sigma_{2 p} \sigma_{2 c}}\left(\sigma_{2 c}-\sigma_{2 p}\right) \sigma_{2}-\sigma_{1 p} \sigma_{1 c}=0
\end{gathered}
$$

where $\sigma_{1 p}, \sigma_{2 p}, \sigma_{1 \mathrm{c}}, \sigma_{2 \mathrm{c}}$-are the tensile strength values along the main reinforcement directions ( $p$-tension, $c$ - compression), and $\tau_{45}$-the tensile strength at pure shear at $\sigma_{1}=\sigma_{1 p}=-\sigma_{2}=-\sigma_{2 c}$.

In this case, the dependence of the ultimate shear strength does not take into account the sign of the shear stress; nonetheless, the Zakharov criterion describes the experimental data satisfactorily.

The simplest representation of the GoldenblattKopnov criterion [8] is a follows: 


$$
\begin{gathered}
\frac{\sigma_{1 c}-\sigma_{1 p}}{2 \sigma_{1 p} \sigma_{1 c}} \sigma_{1}+\frac{\sigma_{2 c}-\sigma_{2 p}}{2 \sigma_{2 p} \sigma_{2 c}} \sigma_{2}+ \\
+\frac{1}{2}\left\{\left(\frac{\sigma_{1 p}+\sigma_{1 c}}{\sigma_{1 p} \sigma_{1 c}}\right) \sigma_{1}^{2}+\left(\frac{\sigma_{2 p}+\sigma_{2 c}}{\sigma_{2 p} \sigma_{2 c}}\right) \sigma_{2}^{2}+\right. \\
+\left[\left(\frac{\sigma_{1 p}+\sigma_{1 c}}{\sigma_{1 p} \sigma_{1 c}}\right)^{2}+\left(\frac{\sigma_{2 p}+\sigma_{2 c}}{\sigma_{2 p} \sigma_{2 c}}\right)^{2}-\right. \\
\left.\left.-\left(\frac{\tau_{45}^{+}+\tau_{45}^{-}}{\tau_{45}^{+} \cdot \tau_{45}^{-}}\right)\right]\right\}^{1 / 2}=1,
\end{gathered}
$$

where $\tau_{45}^{-}$and $\tau_{45}^{+}$- pure shear.

To obtain the five strength constants included in the Zakharov criterion (2), five independent uniaxial elongation, uniaxial compression, and pure shear tests must be performed.

The first and second experiment types according to (2) result in

$$
\left\{\begin{array}{c}
\sigma_{1 p}^{2}+k_{3} \sigma_{1 p}+k_{5}=0 \\
\sigma_{1 c}^{2}+k_{3} \sigma_{1 c}+k_{5}=0
\end{array}\right.
$$

Hence we obtain

$$
k_{3}=\sigma_{1 \mathrm{c}}-\sigma_{1 p} ; k_{5}=-\sigma_{1 p} \sigma_{1 c} .
$$

By performing the third and fourth experiment types, we determine

$$
\left\{\begin{array}{l}
k_{1} \sigma_{2 p}^{2}+k_{4} \sigma_{2 p}+k_{5}=0 \\
k_{1} \sigma_{2 c}^{2}+k_{4} \sigma_{2 c}+k_{5}=0
\end{array}\right.
$$

Hence

$$
k_{1}=\frac{\sigma_{1 p} \sigma_{1 c}}{\sigma_{2 p} \sigma_{2 c}} ; \quad k_{4}=\frac{\sigma_{2 c}-\sigma_{2 p}}{\sigma_{2 p} \sigma_{2 c}} \sigma_{1 p} \cdot \sigma_{1 c} .
$$

The fifth experiment type gives us:

$$
\begin{gathered}
k_{2}=1+\frac{\sigma_{1 p} \sigma_{1 c}}{\sigma_{2 p} \sigma_{2 c}}+\frac{\sigma_{1 c}-\sigma_{1 p}}{\tau_{1}}+ \\
+\frac{\sigma_{1 p} \sigma_{1 c}\left(\sigma_{2 p}-\sigma_{2 c}\right)}{\tau_{1} \sigma_{2 p} \sigma_{2 c}}-\frac{\sigma_{1 c} \sigma_{1 p}}{\tau_{1}^{2}} .
\end{gathered}
$$

As a result of the sixth experiment type, the same constant factor is determined as

$$
\begin{gathered}
k_{2}=1+\frac{\sigma_{1 p} \sigma_{1 c}}{\sigma_{2 p} \sigma_{2 c}}-\frac{\sigma_{1 c}-\sigma_{1 p}}{\tau_{2}}- \\
-\frac{\sigma_{1 p} \sigma_{1 c}\left(\sigma_{2 p}-\sigma_{2 c}\right)}{\tau_{2} \sigma_{2 p} \sigma_{2 c}}-\frac{\sigma_{1 c} \sigma_{1 p}}{\tau_{2}^{2}} .
\end{gathered}
$$

Thus, the Zakharov strength criterion in a plane stress state in a coordinate system that coincides with the material symmetry axes can be represented as

$$
\sigma_{1}^{2}+\frac{\sigma_{1 p} \sigma_{1 c}}{\sigma_{2 p} \sigma_{2 c}} \sigma_{2}^{2}+
$$

$$
\begin{array}{r}
+\left[1+\frac{\sigma_{1 p} \sigma_{1 c}}{\sigma_{2 p} \sigma_{2 c}} \pm \frac{\sigma_{1 c}-\sigma_{1 p}}{\tau_{45}} \pm \frac{\sigma_{1 p} \sigma_{1 c}}{\sigma_{2 p} \sigma_{2 c}}\left(\frac{\sigma_{2 p} \sigma_{2 c}}{\tau_{45}}\right)-\right. \\
\left.-\frac{\sigma_{1 c} \sigma_{1 p}}{\tau_{45}^{2}}\right] \cdot \sigma_{1} \sigma_{2}+\left(\sigma_{1 c}-\sigma_{1 p}\right) \sigma_{1}+ \\
+\frac{\sigma_{1 p} \sigma_{1 c}}{\sigma_{2 p} \sigma_{2 c}}\left(\sigma_{2 c}-\sigma_{2 p}\right) \sigma_{2}-\sigma_{1 p} \sigma_{1 c}=0
\end{array}
$$

where the upper sign is assumed to be + if $\tau_{45}=$ $=\tau_{45}^{+}=\tau_{1}$, and the lower sign is assumed to be - at $\tau_{45}=\tau_{45}^{-}=\tau_{2}$.

Given the Zakharov and Goldenblatt-Kopnov [7] creep-rupture criteria are similar, we can perform necessary strength calculations for a steel barrel using the Zakharov criterion and then compare the results obtained with the results given in [9].

Let us obtain the wall thickness of a closed-end barrel made of low-carbon low-alloy steel with a $0.18 \ldots . .0 .20 \%$ carbon content that is under a uniform internal pressure for a long time according to the operating conditions.

The Goldenblatt-Kopnov strength criterion is written as [8]:

$$
\begin{gathered}
\Pi_{11} \sigma_{11}+\Pi_{22} \sigma_{22}+ \\
+\sqrt{\Pi_{1111} \sigma_{11}^{2}+\Pi_{2222} \sigma_{22}^{2}+2 \Pi_{1122} \sigma_{11} \sigma_{22}}= \\
=\frac{\alpha+\beta e^{-\lambda t_{*}^{-k}}}{n} .
\end{gathered}
$$

where $t_{*}$-durability; $n$ - safety factor; $\alpha, \beta, \lambda, k-$ factors for a given fiberglass laminate.

Let us transform the strength criterion proposed by K. V. Zakharov (3) as follows:

$$
k_{1} \sigma_{1}^{2}+k_{2} \sigma_{2}^{2}+k_{3} \sigma_{1} \sigma_{2}+k_{4} \sigma_{1}+k_{5} \sigma_{2}=1 .
$$

Let us put the factor as

$$
\begin{gathered}
k_{1}=\frac{1}{\sigma_{1 p} \sigma_{1 c}} ; \quad k_{2}=\frac{1}{\sigma_{2 p} \sigma_{2 c}} \\
k_{3}=k_{1}+k_{2}+k_{1} \frac{\sigma_{1 c}-\sigma_{1 p}}{\tau_{45}}-k_{2} \frac{\sigma_{2 c}-\sigma_{2 p}}{\tau_{45}}-\frac{1}{\tau_{45}^{2}} \\
k_{4}=k_{1}\left(\sigma_{1 c}-\sigma_{1 p}\right) \\
k_{5}=k_{2}\left(\sigma_{2 c}-\sigma_{2 p}\right) .
\end{gathered}
$$

where $\sigma_{1 p}, \sigma_{2 p}, \sigma_{1 \mathrm{c}}, \sigma_{2 \mathrm{c}}-$ tensile strength values in the main directions ( $p$-tension, $c-$ compression).

We put the criterion (7) as follows, taking into account the following values of the main stresses

$$
\begin{gathered}
\sigma_{1}=\frac{p R}{2 \delta} ; \quad \sigma_{2}=\frac{p R}{\delta} \\
k_{1}\left(\frac{p R}{\delta}\right)^{2}+\frac{k_{2}}{4}\left(\frac{p R}{\delta}\right)^{2}+\frac{k_{3}}{2}\left(\frac{p R}{\delta}\right)^{2}+k_{4} \frac{p R}{\delta}+k_{5} \frac{p R}{2 \delta}= \\
=\frac{\alpha+\beta e^{-\lambda t_{*}^{-k}}}{n} .
\end{gathered}
$$

*orresponding author: 
where $p$ - pressure; $R$ - radius; and $\delta$ - the pipe wall thickness.

The right side of the equation is replaced using the experimental data obtained, proving the relationship between the short- and long-term tensile strength:

$$
\sigma_{\mathrm{B}}\left(t_{*}\right)=\sigma_{\mathrm{B}}\left(t_{0}\right)\left(\alpha+\beta e^{-\lambda t_{*}^{-k}}\right)
$$

By putting

$$
z=\frac{R}{\delta} ; \quad \frac{\alpha+\beta e^{-\lambda t_{*}^{-k}}}{n}=\bar{n}
$$

we represent (8) as follows:

$$
k_{1} p^{2} z^{2}+\frac{k_{2}}{4} p^{2} z^{2}+\frac{k_{3}}{2} p^{2} z^{2}+k_{4} p z+\frac{k_{5}}{2} p z=\bar{n}
$$

After transforming the equation, we obtain

$$
z^{2}-\alpha z-\text { в }=0,
$$

where

$$
\begin{gathered}
\alpha=\frac{\mathrm{k}_{4}+0,5 \mathrm{k}_{5}}{2\left(\mathrm{k}_{1}+0,25 \mathrm{k}_{2}+0,5 \mathrm{k}_{3}\right) \mathrm{p}} ; \\
\text { B }=\frac{\overline{\mathrm{n}}^{2}}{\left(\mathrm{k}_{1}+0,25 \mathrm{k}_{2}+0,5 \mathrm{k}_{3}\right) \mathrm{p}^{2}} .
\end{gathered}
$$

The real root (9) has the following form:

$$
z=-\alpha+\sqrt{\alpha^{2}+\mathrm{B}} .
$$

The wall thickness of a cylindrical barrel that is $1,020 \mathrm{~mm}$ in diameter with a safety factor of $n=1,11$ will be $\delta=14 \mathrm{~mm}$. Upon comparing the calculation results obtained by the formula (4) with the calculations given in [4], we see that they have only a slight difference.

\section{Conclusions}

The present study leads us to the following conclusions:

1. Experimental studies have shown that the breaking pressure of pipes with internal delamination is within 9.5 $\mathrm{MPa}$. Considering that this value is 2.76 times higher than the operating pressure in the pipeline equal to 3.4 $\mathrm{MPa}$, it can be argued that further long-term operation of the gas pipeline with such defects is possible.

2. It has been proved that instead of cyclic tests for ultimate pressure to determine the remaining life of a gas pipeline, calculation of the minimum permissible wall thickness capable of withstanding the working pressure in the pipeline is sufficient. The data obtained as a result of mathematical calculations prove the correctness of the proposed theory.

3. The results of calculating the wall thickness of a steel barrel, obtained according to two different criteria, coincide with an insignificant error. This makes it possible to use any of the proposed criteria for the strength calculation.
4. The obtained results expand possibilities of applying the considered strength criteria, enabling justifiable selection of methods to operate pipelines, as well as approaches and modes of performing repair and diagnostics and increasing pipe wall strength.

\section{References}

1. V.V. Salyukov, V.V. Kharionovsky, Main gas pipelines. Diagnostics and technical condition management (2016)

ISBN: 978-5-8365-0451-9

2. S.V. Alimov, Gas Industry. 724, 10-15 (2015) [in Russia]

3. V. Linton, E. Gamboa, M. Law, Journal of Pipeline Engineering, 4(1), 207-217 (2007) http://hdl.handle.net/2440/43274

4. A.V. Mitrofanov, N.A. Gafarov, B.V. Kichenko, A.I. Rezvykh, V.A. Polozov, The Science and Research Journal of All-Russia Research Institute of Organisation, Management and Economy in Oil and Gas Industry (NTZh VNIIOENGa), Corrosion prevention and environmental protection, 10, 2-11 (1996) [in Russia]

5. S.A. Bubnov, I.I. Ovchinnikov, A.A. Bubnov, Vestn. Sam. State Tech. University. 2(27), 178-182 (2012) [in Russia]

DOI: https://doi.org/10.14498/vsgtu960

6. M.M. Aliev, R.R. Kantyukov, F.M. Mustafin, K.F. Ulshina, Gas Industry, 12, 56-59 (2015) [in Russia]

7. M.M. Bayburova. Abstract of the of Cand. Sci. (Eng.), Thesis, Saratov, 16 (2008) ) [in Russia]

8. I.I. Goldenblatt, V.A. Kopnov. Izvestia of the Academy of Sciences of the USSR, Mechanics. 6, 77-83 (1965) [in Russia]

9. K.V. Zakharov, Plastics, 8, 61-67 (1961) 